\title{
THE PREVENTION AND TREATMENT OF INFECTIONS BY SPECIFIC IMMUNE THERAPY.*
}

\author{
By EDWARD R. CULLINAN, M.D., F.R.C.P. \\ Assistant Physician, St. Bartholomew's Hospital; Assistant Physician, Woolwich \\ Memorial Hospital.
}

It is the purpose of these two lectures to outline some of the more recent applications of specific immunity to the prevention and treatment of infections. It must be stressed at the outset that these methods are merely adjuncts and should never replace efficient general treatment.

Despite increased knowledge, it must be confessed that there is as yet no real understanding of the whole process of infection and that many of the methods of specific immune therapy, at present in use, are only partly successful. Discouraged by this, many attempts have been made to produce some non-specific substance which will cause an increase of general antibodies against infection, and there are many preparations on the market claiming this to a greater or lesser degree. In the present state of our knowledge, however, it is particularly important to view these claims with the greatest caution. Many of the effects produced by these preparations closely resemble the non-specific reactions brought about by chemical or protein shock, and it is reasonable to believe that the only real advances in immune therapy will continue to be made along specific lines.

\section{Available Methods in Immune Therapy.}

(I) Active immunity.-By injection in a modified form, of organisms living or dead, soluble toxins, or viruses, active immunity can be produced against certain diseases. This is particularly suitable for prophylaxis, as the immunity takes some time to produce, but usually lasts a long time. Good examples of this are vaccination against small pox and inoculation against typhoid fever.

(2) Passive immunity. - Where there is no time to produce active immunity, as in the treatment of acute fevers or of contacts, specific anti-bodies may be injected. The effects of this passive immunity are immediate, but of course, short-lived.

There has been considerable progress in passive immune therapy in the last few years. In addition to tetanus and diphtheria antitoxins, sera containing soluble exotoxins have been made for use in the case of scarlet fever and staphylococcal infections.

Sera containing bacterial antibodies against certain diseases, have been improved.

Human convalescent sera are also being employed to give a brief immunity against measles and poliomyelitis.

In the use of animal sera, there are two dangers. Anaphylaxis needs only to be appreciated to be avoided, and where the danger is suspected the patient may be desensitized. A serum rash, however, which may appear about the eighth day after the injection, is more difficult to prevent, but although it is extremely worrying to the patient, the consequences are seldom serious.

\footnotetext{
-Based on two lectures given under the auspices of the Fellowship of Medicine and Post-Graduate Medical Association, on Ootober 2nd and 3rd, 1933.
} 


\section{Summary of Methods in Use.}

Specific :

Active Immunity.

Vaccines (organisms living or dead).

Soluble exotoxins.

Passive Immunity.

Antibacterial sera.

Antitoxic sera.

i.e., Tetanus.

Diphtheria.

Scarlet fever.

Staphylococcus aureus infection.

Human convalescent sera.

Active and Passive Immunity.

Sensitized vaccines.

Non-Specific.

(Chemical and protein shock, etc.)

Tetanus.

It has long been known that the tetanus bacillus produces an exotoxin, and the method of prevention in cases of deep wounds, by injecting one or two thousande units of antitoxin at an early stage, is well established.

When the disease is fully developed, however, the dose of antitoxin required is very much greater. Two hundred thousand units may be injected intravenously, repeated in twelve hours, and then every twenty-four hours. At the same time eighty thousand units are given intramuscularly, and repeated at first four-hourly, and then in gradually smaller doses with longer intervals between each injection. There is some difference of opinion as to the value of intrathecal injection, but if desired, ten to twenty c.c.'s of concentrated antitoxin may be injected through a lumbar puncture needle after withdrawing a corresponding amount of cerebrospinal fluid.

\section{Diphtheria.}

\section{A.-Test for Susceptibility to Diphtheria (The Schick Test).}

The bacillus of diphtheria also produces an exotoxin. By injecting minute quantities of this exotoxin into the skin, it is possible to determine whether or not an individual is susceptible to the disease. The technique of this, The Schick test, is as follows:-

The flexor surfaces of both fore-arms are cleansed. Fresh toxin is drawn into a tightly fitting syringe, graduated in one-tenths. The needle, with the bevel facing upwards, is inserted into the superficial layers of the skin for half an inch, in such a way that the bevel of the needle is still clearly seen. Two-tenths of a c.c. (I/50 M.L.D.) of the toxin are then injected. The needle is slowly withdrawn and the syringe washed. The opposite arm is treated in exactly the same way with the control fluid consisting of the same toxin which has been heated. The results are read in twenty-four hours, seventy-two hours and ten days. 
There are four possible results.

(I) Positive reaction. A red raised patch appears in the toxin arm in twentyfour to thirty-six hours, reaching its maximum on the third day, and scaling up to ten days.

(2) Negative reaction.

(3) Pseudo-reaction. A red patch appears on both arms on the first day and fades on the third.

(4) Combined pseudo- and positive reactions. On the first day, red patches appear on both arms, but whereas the one on the control arm then begins to fade, the one on the toxin arm increases and reaches its maximum by the third day.

Under the age of three months, only about I5 per cent. of children give a positive reaction, but the figure rises to 60 per cent. in the first year and to 70 per cent. between the first and second years, falling to about 20 per cent. in adults. It may be assumed that the bulk of children up to the age of six are susceptible to the disease.

\section{B.-Prophylaxis.}

The use of toxin, for producing active immunity against diphtheria, is too dangerous, and for some time toxin-antitoxin mixtures were employed. Later a safer preparation was made by mixing toxin with formalin, which was called Anatoxin or Toxoid. Recently, it has been found that the safest and most efficient preparation is a precipitate formed by toxoid and antitoxin, called Toxoid-Antitoxin Floccules (T.A.F.). The dose is adjusted to one c.c. and three injections are given hypodermically, at intervals of one to three weeks. The immunity produced lasts many years.

\section{C.-Treatment of Contacts.}

One to two thousand units of antitoxin may be injected into a person who has been in known contact with diphtheria. This will give passive immunity lasting for two or three weeks, which is a sufficient length of time to prevent the development of the disease.

\section{D. - Treatment of the Disease.}

The disease tends to run a mild course in adults, and in such cases it is sufficient to inject five thousand units of antitoxin on the first or second day. In more severe cases ten thousand units should be given and repeated in twelve hours. In very severe cases, or whenever the larynx is involved, the initial dose should be twenty thousand units, which should be repeated. Injections may be intramuscular or subcutaneous. In very severe cases, when an immediate effect is desired, the intravenous route should be chosen.

\section{E.-Treatment of Carriers.}

Many of the diphtheria bacilli found in the throats of apparently healthy people are non-virulent, and it is well to decide this point before undertaking any vigorous measures for their eradication. This is done by culturing the organisms and injecting them into a guinea-pig. 
Carriers of virulent bacilli are a public menace, and are unfortunately very difficult to treat. They should be isolated and given as much fresh air as possible. Specific therapy is seldom of value, although vaccines of diphtheria bacilli may be tried. These vaccines, either stock or autogenous, may be injected in doses of five to ten million every six days. The treatment usually resolves itself, however, into dealing with the rhinitis or removing the tonsils.

\section{Staphylococcal Infections.}

Active immunisation with staphylococcal vaccines has for many years been widely used with varying results. Also, for the treatment of generalised staphylococcal infections an antibacterial serum has been available, but the results have been disappointing. It has recently been shown, however, that the Staphylococcus aureus produces an exotoxin and this has been isolated. The toxin is rapidly converted by means of formalin into toxoid, and researches are taking place to determine the value of this toxoid in producing active immunity against staphylococcal infections. A concentrated antitoxin has also been prepared for the treatment of cases of staphylococcal infection. Although the use of this serum is still in the experimental stage, the results so far obtained are promising. In cases of staphylococcal septicæmia the antitoxin should be given early, intravenously, and in large doses.

The necessity for the surgical treatment of any local focus of infection of course remains.

\section{Scarlet Fever.}

The streptococcus found in the throats of cases of scarlet fever produces an erythrogenic exotoxin. The isolation of this toxin has made it possible both to test the susceptibility of individuals to scarlet fever, and to immunise them against it. Moreover, the preparation of an antitoxin has also furthered the treatment of the disease.

\section{A.-Test for Susceptibility to Scarlet Fever (The Dick Test).}

Using the same technique as in the Schick test, I/Io c.c. of scarlet fever toxin is injected intradermally into one arm and I/Io c.c. of control fluid (i.e. the heated toxin) into the other. The result is read in 4, I2, 24, and 48 hours, the important time being 24 hours. As in the Schick test, four results are possible.

(I) Positive reaction. This appears in the toxin arm as early as 4-6 hours and reaches its maximum in I2 hours. After 24 hours, three grades of the reaction may be seen, according to the lack of immunity present.

(a) Strong positive. Red tender swelling, with a sharp edge 3-7 cms. diam. After 48 hours the swelling fades and there is fine desquammation on the seventh to the tenth day.

(b) Positive. Red tender swelling, I.5-3 cms. diam.

(c) Slight positive. Faint red mark, less than $2 \mathrm{cms}$. diam.

(2) Negative reaction. 
(3) Pseudo-reaction. The same result in both arms. The patches fade more quickly than in the positive reaction.

(4) Combined pseudo- and positive reactions.

Under the age of six months about 45 per cent. of children give a positive reaction, rising to about 70 per cent. between the ages of one and two, and gradually falling again towards adult life.

\section{B.-The Dick Test in a Suspected Case of Scarlet Fever.}

In the first day or so, a negative Dick test is evidence against the disease. A strongly positive Dick test after the third day of the illness is also evidence against scarlet fever.

\section{C.-Prophylaxis. Active Immunity by Means of the Soluble Exotoxin.}

The toxin consists of a filtrate of a special hæmolytic streptococcus. Four injections are given to a susceptible individual at weekly intervals intramuscularly or subcutaneously. The doses are 500, I,000, 5,000, and Io,000 "skin test doses".

These injections are sometimes followed by a local reaction, a sore throat, or even a rash. It is hoped that in the near future the toxin will be replaced by anatoxin, or a toxoid-antitoxin mixture, similar to Diphtheria T.A.F., and that then the occasional unpleasant effects will be avoided. After the prophylactic inoculations have been given, the Dick test is done again. If the result is negative it is to be assumed that immunity has been established. It is not yet known how long this immunity lasts, but it certainly does so for many years.

\section{D.-Treatment of Contacts.}

If the Dick test is positive in a person who has been in known contact with scarlet fever, an intramuscular injection of five c.c.'s of concentrated scarlatinal antitoxin, will give passive immunity lasting from ten to fourteen days.

\section{E.-Treatment of the Disease.}

If in the early stages of a suspected case a hæmolytic streptococcus is found in the throat and the Dick test is positive, ten c.c.'s of concentrated scarlatinal antitoxin should be injected. If, after 24-36 hours the temperature and pulse rate are still raised, a further Io c.c.'s of antitoxin should be given. Antitoxin, which must be administered early, reduces the temperature and relieves the symptoms, but it is doubtful whether it has any effect in preventing septic complications. Recently a dual serum has been used, consisting of antitoxin and antibacterial serum. It has been suggested that human serum from a convalescent scarlet fever case should be used for treatment when available. This would be given intravenously and has the advantage that there is no danger of anaphylaxis. In a very severe case, where there are hæmorrhages, a blood transfusion may be given from a donor who is convalescent from scarlet fever.

\section{Other Streptococcal Infections.}

One of the modern views of streptococci is that they belong to one race but are capable of producing different effects. This theory would explain why scarlatinal antitoxin is completely successful in cases of scarlet fever but only partly so in cases of other streptococcal infections. 


\section{Erysipelas.}

50 c.c.'s of univalent streptococcal pyogenes antibacterial serum is injected intravenously, and this may be repeated. The serum has recently been concentrated, and may be given in smaller amounts. It is also advisable to carry out the Dick test and if this is positive, concentrated scarlatinal antitoxic serum may also be given.

\section{Streptococcal Septicæmia.}

The present position of immune therapy in the treatment of blood stream infections by the Streptococcus pyogenes is still rather unsatisfactory. Antibacterial sera should be given in generous quantities and also scarlatinal antitoxic serum. The latter is thought to be especially useful in cases of puerperal septicæmia, and is of undoubted value in cases associated with a tonsillitis. 20 c.c.'s may be given intravenously, at repeated intervals.

Although not related to immune therapy, a word should be said about the use of antiseptics. Every type of antiseptic has been tried in an effort to kill the organisms in the blood stream; aniline dyes, cinchona, hypochlorites, arsenic, mercury, silver, antimony, phenols, and others but up to the present none has proved of the slightest value in the treatment of this disease.

In severe cases, after other methods have been tried without success, sensitised

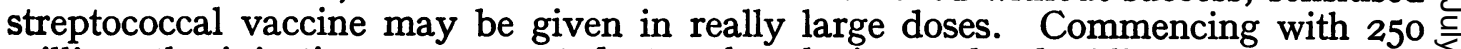
million, the injections are repeated at I2-hourly intervals, doubling the dose each time until 8,000 million is reached. Remarkably good results are sometimes obtained from this somewhat drastic procedure.

\section{Lobar Pneumonia.}

\section{Pneumonia.}

Pneumococci belong to four main groups. Type IV. is a heterogeneous group, and includes the normal inhabitants of the upper respiratory passages. Types I. and II. are not normally found in the mouth and appear to be the causal organisms in 60 per cent. of cases of lobar pneumonia. A concentrated antibacterial serum, containing antibodies to types one and two has been prepared for the treatment of pneumonia by Felton and more recently, sera for the separate types have been put up in this country.

In order to avoid giving serum unnecessarily, the type of pneumococcus present in the sputum should be ascertained. This can be done by one of two methods:-

(I) Sabin. The sputum is rubbed in sterile broth and injected into the peritoneum of a mouse. Six hours later the peritoneal exudate is withdrawn and a drop placed on a slide, together with a drop of Type I. antiserum. After being allowed to stand for two minutes this is dried, stained and examined under the microscope. If the organism belongs to Type I. the cocci will be seen to have clumped together. If there is no clumping the test is repeated by mixing a drop ${ }^{N}$ of the exudate with a drop of Type II. antiserum and the effect observed.

(2) Armstrong. If the sputum is thin and pneumococci are present in large numbers, the test can be carried out with the fresh sputum. In this case $\frac{}{\Phi}$ it is unnecessary to stain. With the appropriate serum the capsules of the organisms $\stackrel{?}{+}$ swell up.

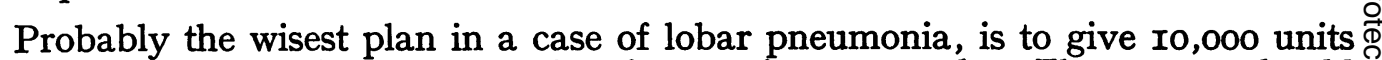
each of Types I. and II. concentrated antiserum, intravenously. The sputum should $\stackrel{\odot}{\circ}$ 
then be typed, and if the organisms belong to Types I. or II., ten thousand units more serum of the appropriate variety may be given at eight hourly intervals. Alternatively fifty thousand to one hundred thousand units may be given in the first 24 hours and repeated if necessary. Of course, if the organisms belong to Types III. or IV., the serum should be discontinued.

This serum seems to modify the clinical course of the disease, precipitate the crisis and lower the mortality, but it is very doubtful whether it lessens the liability to complications. The treatment is unsuitable for cases of bronchopneumonia, which are usually associated with Type IV. pneumococci, and also of influenzal pneumonia.

\section{Treatment.}

\section{Cerebro-Spinal Fever.}

There are four types of meningococci, and antibacterial sera have been made for each type. It is desirable, therefore, to type the organisms in any given case in order that the appropriate serum may be employed. As this is seldom possible, a polyvalent serum is most frequently used. The method of giving it is as follows. Cerebro-spinal fluid is drawn off through a lumbar puncture needle and the amount measured. The serum, warmed to body temperature, is then injected very slowly. This is most conveniently done with a record syringe, but the speed of injection should not be greater than I c.c. per minute. The method of running it in by gravity through a funnel is usually unsatisfactory. The amount of serum should be about 5 c.c.'s less than the amount of cerebro-spinal fluid withdrawn. The injection is repeated daily during the first week, and on alternate days during the second week. Further injections depend on the course of the disease.

It seems probable that in time to come an antitoxic serum may also be available for the treatment of the disease.

\section{Anthrax.}

The essential points in the treatment of anthrax consist in starting specific treatment as early as possible, and in not interfering with the pustule, which should on no account be cauterised or incised. The pustule is best treated with a mild antiseptic and the affected limb put at rest. Large doses of anti-anthrax serum should be given at once, commencing with 40 c.c.'s intravenously, followed in I2-24 hours by 30 c.c.'s intramuscularly. If anti-anthrax serum is not immediately available, 0.6 to 0.9 grammes of N.A.B. should be given intravenously, and, if necessary, repeated on the following day. It is claimed that this treatment with arseno-benzol is curative.

\section{Gonococcal Infections.}

No effective antiserum has yet been prepared for the treatment of this infection and vaccines are useless for prophylaxis. The vaccine, however, given in minute doses is sometimes of value in cases of gonococcal arthritis.

\section{Tuberculosis.}

\section{A.-Prophylaxis.}

Experiments, on a large scale, are being made in France to produce active immunity to tuberculosis in young children. A strain of the tubercle 
bacillus, which has been grown since I908, has become so attenuated that it is said to be innocuous to a guinea-pig on injection. This bacillus is called the Bacille Calmette-Guérin (B.C.G.). Three doses of the living bacilli are given, by mouth, to infants during the first ten days of life. The method is not in use in this country, and an insufficient number of years has passed to judge the results of this prophylactic attempt. There are certain theoretical objections to the method; firstly, that the dose of bacilli is unmeasurable, and secondly, that such attenuated organisms might conceivably regain their virulence in the culture medium of the human body.

\section{B.-Treatment.}

No satisfactory serum has yet been devised for the treatment of tuberculosis. In cases of genital tubercle, however, Koch's old tuberculin is of value. It is essential that this treatment should be leisurely. If a lung lesion is also present, the initial dose should be I/500,000 of a milligramme, the following doses at four day intervals being: a four hundred thousandth, a three hundred thousandth, a two hundred thousandth, a hundred thousandth, a seventy-fifth thousandth, a sixty thousandth, a forty-fifth thousandth, and so on up to a ten thousandth and then at seven day intervals up to a five hundredth of a milligramme. If there is no lung lesion, the initial dose may be a ten thousandth working up very gradually at weekly intervals to a thousandth of a milligramme.

\section{Prophylaxis.}

\section{Whooping Cough.}

The Bacillus pertussis vaccine deserves wider recognition. It has no proven value in the treatment of the disease but is well worth using for prophylaxis.

\section{Convalescent Human Sera.}

Convalescent human sera are being used in certain diseases, thought to be due to viruses. They have been tried without success in mumps and chicken-pox. Researches are at present being made as to their value in poliomyelitis, but the results are difficult to assess as the serum is said to be of use only in the preparalytic stage, when the criteria of diagnosis are uncertain. Encouraging results have been reported in the treatment of cases of post-vaccinal encephalitis.

\section{Measles.}

Injection of human serum from a recently convalescent case of measles will prevent the disease in a contact, but being passive, the immunity will only last for two or three weeks. It is well known that an attack of measles will usually confer a life-long active immunity, and if convalescent serum is not available, the serum of an adult who has had measles in childhood will suffice. The dose of serum is 3 to I2 c.c.'s from a convalescent case or 6 to 24 c.c.'s from an adult. If serum cannot be prepared, even whole blood may be used, injected with a paraffined syringe. If the measles contact is under the age of three, the disease should be prevented by giving the serum as soon as possible during the first five days after exposure. If the child is older than three years it is better to give the serum between the sixth and ninth days after contact. This will modify the course but not prevent the disease. However, the child will have only a mild attack of measles and thereby gain a lasting immunity against further infection. 